\title{
Morphological Characteristics of The Arterial Supply of The Extra- Hepatic Biliary System
}

\author{
R.Hasan ${ }^{1}$, V.Abeysuriya ${ }^{2}$, S.Kumarage ${ }^{3}$, J.A.A.S. Wijesinghe ${ }^{4}$ \\ ${ }^{l}$ Department Of Anatomy, Faculty Of Medicine, University Of Kelaniya, Sri Lanka \\ ${ }^{2}$ Department Of Anatomy, Faculty Of Medicine, University Of Kelaniya, Sri Lanka \\ ${ }^{3}$ Department Of Surgery, Faculty Of Medicine, University Of Kelaniya, Sri Lanka \\ ${ }^{4}$ Faculty Of Medicine, University Of Kelaniya, Sri Lanka
}

\begin{abstract}
:
Introduction: A comprehensive knowledge of the regional anatomy and abnormalities of the arterial supply to the extra- hepatic biliary system is a requisite for safe biliary tract surgery.

Objective: To describe morphological characteristics of arterial supply to extra-hepatic biliary system using human cadavers.

Method: Descriptive-prospective cross sectional study was performed in 60 cadavers, to observe variations in arterial supply to extra- hepatic biliary system.

Results: The mean length of right and left hepatic arteries was $2.8 \mathrm{~cm}$ and $2.3 \mathrm{~cm}$ respectively. The mean length of proper hepatic artery was $3.1 \mathrm{~cm}$. The mean length of cystic artery was $1.4 \mathrm{~cm}$ before its division into anterior and posterior branches ranged from $0.9 \mathrm{~cm}$ to $2.1 \mathrm{~cm} .74 .9 \%$ of the cystic arteries originated as a single artery from the right branch of the hepatic artery. In $43 \%$ of the specimens, right hepatic artery was lying in the triangle of Calot's.
\end{abstract}

Conclusion: Variations in arterial supply inrelation to the extra-hepatic biliary system vasculature is common.

\section{Introduction}

Comprehensive knowledge in anatomy of the biliary system and the anatomical variations encountered in the general population is an essential prerequisite for Cholecystectomy. In laparoscopic cholecystectomy, the surgeon must have a knowledge on distortions of regional anatomyand vasculature as this altersthe site and the direction of retraction according to the spatial relationships of the region. This regional anatomical variations of vasculaturehavesignificant importance as it results in technical difficulties and incidental iatrogenic damage in their presence. ${ }^{1}$.

\section{Arterial supply to extra- hepatic biliary system}

The cystic artery arises from the right branch of the hepatic artery and may pass anterior or posterior to the common hepatic duct, in the left border of the triangle of Calot ${ }^{2,3,4}$. The boundaries of Calot's triangle are the inferior surface of the right lobe of the liver (upper boundary) and the cystic duct (lower boundary) and the common hepatic duct (left border). The Calot's triangle contains the cystic artery, often the right hepatic artery and occasionally a bile duct and is of key significance during dissection for a Cholecystectomy ${ }^{5}$

\section{Biliary vascular anomalies}

The blood supply to the gallbladder is via the cystic artery which has multiple and often common variations. The typical course that it arises from the right hepatic artery and lies in the Calot's triangle. The cystic artery may arise from the common or left hepatic artery and may course anterior or posterior to the common hepatic duct. Occasionally the cystic artery arises as a branch of the gastroduodenal artery ${ }^{1,4,6}$

\section{Objective}

To describe the morphological characteristics of the arterial supply to extra- hepatic biliary system using human cadavers.

\section{Methodology}

A descriptive-prospective cross sectional study was carried out in 60 cadavers in the Department of Anatomy, Faculty of Medicine, University of Kelaniya. The principle investigator did the cadaveric dissections and the findings were confirmed by a senior anatomist. Data was analyzed using Statistical Package for Social Sciences 11(SPSS). 
The anterior abdominal wall was cut open longitudinally along the midline. The abdominal contents were separated on to the right side along the central margin up to the mid axillary line. Thereafter the abdominal wall was divided from the right side of the pubic bone up to the anterior superior iliac spine. The anterior abdominal wall flap was reflected laterally. Stomach was retracted to the left side and second part of the duodenum, free margin of the lesser Omentum, epiploic foramen and gall bladder identified. Dissection was done to demonstrate the extra hepatic biliary system and its vascular pattern. Specimens were studied and photographed, especially the variations. Length of the left and the right hepatic arteries, from the origin from the proper hepatic artery to entry point of the liverwere measured using a caliper.

\section{Results}

Arterial supply of the extra-hepatic biliary system (Table 1)

The mean lengths of right and left hepatic arteries were $2.8 \mathrm{~cm}$ and $2.3 \mathrm{~cm}$ ranging from $2.0 \mathrm{~cm}$ to $5.5 \mathrm{~cm}$ and $1.0 \mathrm{~cm}$ and $4.2 \mathrm{~cm}$ respectively. The mean length of proper hepatic artery was $3.1 \mathrm{~cm}$ ranging from $1.8 \mathrm{~cm}$ to $6.8 \mathrm{~cm}$. The mean length of cystic artery was $1.4 \mathrm{c} \mathrm{m}$ before its division in to anterior and posterior branches with a range of $0.9 \mathrm{~cm}$ to $2.1 \mathrm{~cm}$.

Seventy-five percent of the cystic arteries originated as a single artery from the right branch of the hepatic artery and passed posterior to the common hepatic duct, in the left border of the triangle of Calot and divided in to anterior and posterior branches near the body of the gall bladder. Twelve percent had two cystic arteries originating separately from right hepatic artery. Five percent of cystic arteries passed anterior to the common hepatic artery and $1.7 \%$ traversed over common bile duct while $1.7 \%$ passed on the cystic duct itself. The origins of cystic artery from gastro duodenal artery or from proper hepatic artery that originated from the abdominal aorta were seen in $1.7 \%$ each. Forty-three percent of the specimens right hepatic artery was lying in the triangle of Calot's. One specimen was found to have the right hepatic artery on the common bile duct. Origin of the cystic artery from the left hepatic artery or right hepatic artery crossing anterior to common hepatic duct or right hepatic artery, while traveling posterior to the portal vein were not noted.

\section{Discussion}

Most of the classical textbooks describe the common or normal, morphological characteristics and some of the anomalies arterial supply to the extra hepatic biliary system ${ }^{4}$.However, the incidence of occurrence of common and abnormal anatomical characteristics was not comprehensively described. Also most textbooks were based on western data and lacked a comprehensive description of this important regional anatomy with relevance to a geographic regional variation, especially in the context of south East Asia.

There is no literature to compare the regional variation of morphometry of the hepatic arteries and the cystic artery. Available literature ${ }^{2}$ revealed $75.5 \%$ of the cystic arteries originated as a single artery from the right branch of the hepatic artery and passed posterior to the common hepatic duct, in the left border of the triangle of Calot and divided in to anterior and posterior branches near the body of the gall bladder, where as in our study it was $74.9 \%$. The incidence of the cystic artery originates from the right hepatic, the proper hepatic or from the gastro duodenal artery and traverse anterior to the common hepatic duct was $13.1 \%, 2.1 \%$ and $2.6 \%$ respectively ${ }^{3}$. In our study $5 \%$ of cystic arteries passed anterior to the common hepatic duct and $1.7 \%$ traversed over the common bile duct and $1.7 \%$ passed on the cystic duct itself. The origins of the cystic artery from the gastro duodenal artery or from the proper hepatic artery that originated from the abdominal aorta were seen in $1.7 \%$ each. Fifty-three of the specimens the right hepatic artery was lying in the triangle of Calot's. Origin of the cystic artery from the left hepatic artery or the right hepatic artery crossing anterior to common hepatic duct or from the right hepatic artery, while traveling posterior to the portal vein were not noted

\section{Conclusions}

Abnormalities in arterial supply to extra- hepatic biliary system are common. Presences of rare anomalies are not infrequent. The awareness of variations in the related vascular pattern is essential in preventing iatrogenic injury to vascular system during cholecystectomy especially in laparoscopic cholecystectomy.

Table 1: Morphometry of the arterial supply to Extra-hepatic biliary system.

\begin{tabular}{|l|c|c|}
\hline \multicolumn{1}{|c|}{ Artery } & Range of length & Mean length \\
\hline Right hepatic artery & $2.0 \mathrm{~cm}-5.5 \mathrm{~cm}$ & $2.8 \mathrm{~cm}$ \\
\hline Left hepatic artery & $1.0 \mathrm{~cm}-4.2 \mathrm{~cm}$ & $2.3 \mathrm{~cm}$ \\
\hline Proper hepatic artery & $1.8 \mathrm{~cm}-6.8 \mathrm{~cm}$ & $3.1 \mathrm{~cm}$ \\
\hline Cystic artery & $0.9 \mathrm{~cm}-2.1 \mathrm{~cm}$ & $1.4 \mathrm{~cm}$ \\
\hline
\end{tabular}




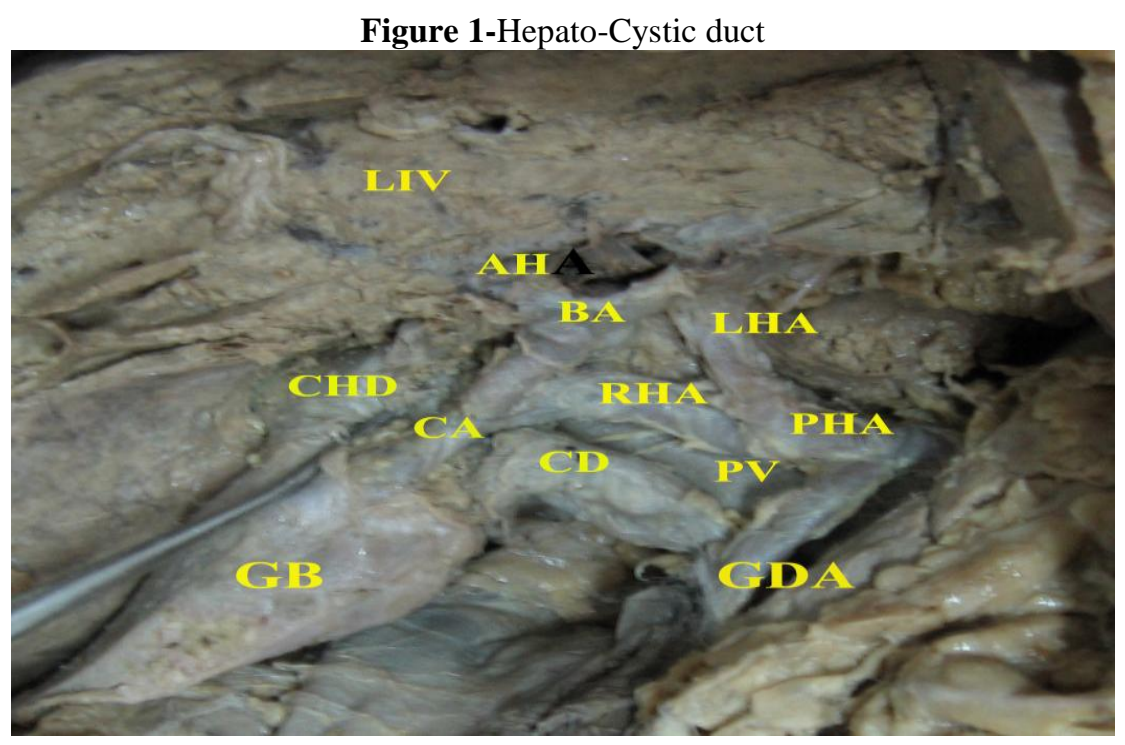

Hepato-Cystic duct illustration:Liver (LIV) with partially dissected right lobe is seen cranially. The common hepatic duct (CHD) directly drains to the upper segment of the gallbladder (GB). From the GB the cystic duct (CD) originates and drains out as the common bile duct without joining to the CHD. The common hepatic artery is divided into the proper hepatic artery (PHA) and the gastro-duodenal artery (GDA). The portal vein (PV) is seen between the CD and the PHA. The PHA divides into left and right hepatic arteries (LHA \&RHA). The cystic artery (CA) is originating from the RHA. A "bridging artery" (BA) connects the right and left hepatic arteries. An accessory hepatic artery $(\mathrm{AH})$ is originating from the bridging artery, forming a "cruciate" hepatic arterial anastomosis.

\section{References}

[1]. Chummy S. Sinnatamby (2000), Last's Anatomy, Regional and Applied, 13th edtion, Churchill Livingstone, UK, page 257-260

[2]. Chaurasia, B.D. (1995). Lower limb and Abdomen. Human Anatomy. Volume two $3^{\text {rd }}$ edn, New Delhi, CBS publishers and distributors, page 237-42,

[3]. Anne M, Agur R, Ming J. Lee Grants (1999). Abdomen, Atlas of Anatomy, 09th edn, Lippincot Williams \& Wilkins publishers and distributers. pp 118-120

[4]. Lamah M, Karanjia ND, Dickson GH. Anatomical variations of the extrahepatic biliary tree: review of the world literature. Clin Anat. 2002; 15(4): 314-5

[5]. Champetier J, Letoublon C, Arvieux C, Gerard P, Labrosse PA. Variations of division of the extrahepatic bile ducts: significance and origin, surgical implications.JChir $1989 ; 126(3): 147-54$

[6]. Prinz RA, Howell HS, Pickleman JR Surgical significance of extrahepatic biliary tree anomalies. Am J Surg. 1976; 131(6): 755-7 